bar erschien, als der Urnebel bereits eine Struktur besaß. So nahm er an, daß Sonne und Planeten in einem streifenförmigen und leicht gebogenen Nebel keimartig vorgebildet waren, aus dem sie sich als Einzelkörper weiterentwickelten, als der Zerfall der Nebelmaterie in Teilmassen begann. Planeten und Satelliten folgten getrennten Entwicklungswegen, während die Kometen wahrscheinlich erst später als örtliche Verdichtungen eines von der Sonne durchschrittenen Nebels eingefangen wurden.

Auf diese Weise ist Nölke in zahlreichen Veröffentlichungen mit unbeirrbarer Kritik den sich ihm aufdrängenden Problemen nachgegangen. Mit erstaun licher Umsicht und Sachkenntnis versuchte er, die Probleme der kosmischen Entwicklung, das Wesen der Nebel, Novae, Doppelsterne und $\delta$-Cephei-Sterne sowie die kosmischen Ursachen der irdischen Eiszeiten zu erhellen, um sich darüber hinaus mit ebenso großer Sorgfalt mit Fragen der Störungsgleichungen, Welteislehre, Wegenerschen Verschiebungstheorie und Geotektonik auseinanderzusetzen. Die in vielen Einzel- untersuchungen vorliegenden Ergebnisse faßte er alsdann zu seinem eigentlichen Hauptwerk „Der Entwicklungsgang unseres Planetensystems“ (erschienen in drei Auflagen 1908, 1919 und 1930) zusammen, das von den beiden Büchern „Entwicklung im Weltall“ (1926) und „Geotektonische Hypothesen“ (1924) ergänzt wird. Die letztgenannten Schriften liegen auch in spanischer Übersetzung vor.

Nölkes Arbeiten zeichnen sich durch pädagogische Klarheit aus. Kritik bedeutete ihm Suchen nach der Wahrheit, wobei er sich der Grenzen wissenschaftlicher Erkenntnis vollauf bewußt blieb, und dort, wo Antworten nicht mehr zu erwarten waren, in Selbstbescheidung nicht weiterfragte. Ein unglücklicher Tod hat diesem reichen Leben ein schnelles Ziel gesetzt. Um ihn trauern zahlreiche Schüler und Freunde, in schmerzlicher Bewegung aber seine Familie, deren in seltener Harmonie gefügtes Band durch einen harten Schicksalsspruch so jäh zerrissen ward.

Diedrich Wattenberg (Bremen).

\title{
BESPRECHUNGEN
}

Die Herkunft der Sterne von Pascua l Jordan. Wissenschaftl. Verlagsgesellschaft m.b.H. Stutt gart 1947. 70 S., $8^{\circ}$, Preis RM 3.-.

Das Werk ist in der Hauptsache eine Zusammenfassung neuerer Arbeiten des Verfassers, die sich auf die folgenden drei Gegenstände beziehen: 1. Die Entstehung der Sterne, 2. Entartete Sterne, 3. Veränderliche Sterne.

Der erste Abschnitt des Buches fußt auf einer von D i r a c entwickelten Vorstellung (von Jordan Diracsches Prinzip genannt), welche besagt, dàß alle aus echten Naturkonstanten gebildeten dimensionslosen Zahlen von der Größenordnung 1 (allerdings sehr weit gefaßt) sein sollten. Beispiel: $h c / e^{2}=861$, dagegen $e^{2} / m_{P} m_{e} f$, das Verhältnis der Coulombkraft zwischen Proton und Elektron zur Gravitation, $10^{40}$. Dirac hat versucht, plausibel zu machen, daß Zahlen von dieser Größenordnung keine wirklichen Konstanten, sondern Funktionen des Weltalters sind. $\mathrm{Ob}$ dieser Versuch als geglückt anzusehen ist, wird von Jordan nicht zur Debatte gestellt, jedenfalls unternimmt er es, die kosmologischen Folgerungen aus diesem Prinzip zu entwickeln und sie mit der Erfahrung $\mathrm{zu}$ vergleichen.

Die Makrophysik liefert sechs wesentliche empirische Größen: Die Lichtgeschwindigkeit $\boldsymbol{c}$, die Gravitationskonstante $f=\chi c^{2} / 8 \pi$, das Weltalter $A$, die Hubble-Konstante $a\left[\mathrm{sec}^{-1}\right]$, den Krümmungsradius der Welt $R$ (abgeleitet aus Sternzählungen) und die mittlere Massendichte im Kosmos $\mu$. Es zeigt sich nun, daß folgende Beziehungen annähernd gelten:

$$
a^{-1} \approx A, \quad R \approx c A, \quad \mu^{-1} \approx f A^{2} .
$$

Die ersten beiden Beziehungen lassen sich auf Grund der Vorstellung der Expansion der Welt sofort an- schaulich verstehen. Die dritte führt dann aber (wenn sie kein Zufall ist) $\mathrm{zu}$ der Folgerung, daß $f$ und die Masse der Welt $M\left(\mu R^{3}\right)$ nicht konstant, sondern Funktionen des Weltalters sind. Im Bereich der Mikrophysik definieren ferner die Konstanten $h, c$ und die Elementarlänge $l=2 \cdot 10^{-13}$ eine Masseneinheit von annähernd dem Betrage der Mesonenmasse $\boldsymbol{m}_{\boldsymbol{M}}$ und eine Elementarzeit $\tau=l / c=10^{-23}$ sec. In dieser Einheit gilt ungefähr

$$
\begin{aligned}
R / l \approx \sqrt{M / m_{M}} & \approx \\
& \approx m_{M} / l \\
& \approx \gamma(\text { dimensionslose Zahl }) \approx A / \tau .
\end{aligned}
$$

Nach D i r a c sind diese Beziehungen kein Zufall, sondern es ist $\gamma$ eben das Maß für das Weltalter; damit wird die Weltmasse proportional $\gamma^{2}$ und die Gra-vitationskonstante $\sim \mathbf{1} / \gamma$. Die Massen der größten Sterne sind nur von der Ordnung $\gamma^{3 / 2} m_{P}$. Dies deutet Jordan durch die Annahme, daß Sterne mit umso größerer Masse entstanden sind, je jünger sie sind, und zwar ist die Vorstellung die, daß die Sterne mit etwa Kerndichte plötzlich entstehen (dies kann, wie Jordan zeigt, energetisch kostenfrei geschehen) und daß dann der Stern sich auf normale Dichte ausdehnt. Den Vorgang der Materiebildung und Sternentstehung identifiziert Jordan, in Anlehnung an einen Gedanken von Unsöld, mit dem Aufleuchten der Supernovae, bei dem er sich auch die Höhenstrahlung entstanden vorstellt. - Die Zahl der Spiralnebel ist von der Ordnung $\gamma^{1 / 4}$, es müssen sich also auch Spiralnebel ständig neu bilden. Extrapoliert man die Geschichte des Kosmos gemäß diesem Bilde nach rückwärts, so kommt man zu der Folgerung, daß zur Zeit $\tau=10^{-23}$ sec nach der Entstehung der Welt der Kosmos den Radius $l$ besaß und ein Neutronenpaar enthielt, bei gegenseiti- 
ger Kompensation von Gravitationsenergie und Ruheenergie.

Der zweite Abschnitt ist im Gegensatz zum ersten durchaus nicht spekulativ. Er behandelt die Auflösung einer Schwierigkeit der bisherigen Theorie des Aufbaus, daß nämlich im Rahmen dieser Theorie kein Endzustand hoher Dichte für Sterne oberhalb von etwa $1^{1} / 2$ Sonnenmassen erkennbar war. Jordan zeigt, daß die Berücksichtigung des (an sich schon bekannten) Umstandes, daß bei Dichten oberhalb von $10^{34}\left[\mathrm{~cm}^{-3}\right]$ $\alpha$-Teilchen und Elektronen spontan in Neutronen übergehen, die Schwierigkeit behebt. Sterne oberhalb der Grenzmasse entwickeln also einen Neutronenkern.

Der letzte Abschnitt geht aus von Beobachtungen Guthnicks an speziellen, regelmäßig veränderlichen Sternen, welche dahin gedeutet werden können, daß im Sterninnern ein Kern (oder auch zwei einander umkreisende Kerne) pulsieren. Ob die von diesen Pulsationen ausgehenden Druckwellen die Oberfläche erreichen, hängt von dem Zustand der Gashülle ab. Zur Deutung der beobachteten Eigenschaften der Veränderlichen wird Gebrauch gemacht von der Vorstellung, daß ein Gemisch von Ionen, Elektronen und Neutronen in bestimmten Zustandsbereichen die Eigenschaft besitzt, sich bei adiabatischer Ausdehnung zu erhitzen, so daß ein derartiger Kern vielleicht ähnlich wie ein Explosionsmotor arbeiten könnte. - Zum Schluß werden hinsichtlich des Aufbaus der Riesensterne und der Neuen Sterne folgende Modellvorstellungen vorgeschlagen: Den Riesen schreibt Jordan einen sich kontinuierlich vergrößernden pulsierenden Neutronenkern zu, der im Falle von Resonanz mit der Hülle auf Veränderlichkeit der Sternhelligkeit führt; die Energiequelle dieser Sterne ist die Gravitationsenergie des Kerns. Die Energiequelle der Neuen Sterne dagegen wäre der Weizsäcker-Bethe-Zyklus in einer noch Wasserstoff enthaltenden äußeren Hülle dieser sehr dichten Sterne.

Referent möchte den Wert des Werkes hauptsächlich in der Fülle der Anregungen sehen, die den belohnt, der allen Gedankengängen des Verfassers wirklich nachgeht.

L u d w ig B i ermann.

\section{Deutscher Wetterdienst in der US-Zone}

Durch eine Vereinbarung der Ministerpräsidenten der drei Länder der amerikanischen Besatzungszone vom 3. Dezember 1946, der sich inzwischen das Land Bremen angeschlossen hat, ist der Deutsche Wet terdienst in der US-Z on e als Körperschaft des öffentlichen Rechts gegründet worden. Er umfaßt ein Zentralamt in Bad Kissingen, Postfach 50, den Wetterdienst München, die Ämter für Wetterdienst in Kassel, Frankfurt a. M., Karlsruhe, Nürnberg-Fürth, Stuttgart. die Agrarmeteorologischen Versuchsstationen
Geisenheim und Gießen, die Bioklimatischen Stationen Königstein (Taunus), Oberstdorf und Bad Tölz, 3 Flugwetterwarten, 10 Bergstationen, 32 zusätzliche Meldestellen des synoptischen Meldedienstes, 155 Klimastationen, 1244 Niederschlagsmeßstellen und 1190 phänologische Beobachter. Am Zentralamt besteht neben der synoptischen Abteilung, die die Wettervorhersage zu ihrer Aufgabe hat und dabei insbesondere die Belange der Wirtschaft im Auge behält, eine klimatologische Abteilung, in der die vielfachen Beziehungen des Wetters zum Leben verfolgt werden, und u. a. eine Bibliothek mit rund 50000 Bänden meteorologischer Fachliteratur, die größte derartige Bibliothek in Deutschland. Auch das alte deutsche Beobachtungsmaterial ist in umfangreichen Archiven dort zum größten Teil noch vorhanden.

Das Amt und seine Außenstellen sind zu allen Auskünften über meteorologische Dinge jederzeit bereit.

\section{NACHRICHTEN}

Am 26. November 1947 wurde als Vorstufe für eine „Deutsche Geophysikalische Gesellschaft“ die „G e o physikalische Gesellschaft in $\mathrm{Ham}$ b u r g" gegründet. Anschrift: Geophysikalisches Institut der Universität, Hamburg 13, Rothenbaumchaussee 33 .

\section{B E R I C H T I G U N G E N}

Berichtigungen zu A. Münster, Über einige Eigenschaften gelöster Fadenmoleküle, III. Mitt.: Die Solvatation der Fadenmoleküle (Z. Naturforschg. 2a, 284 [1947]).

S. 290, Gl. (29): Der erste Faktor der rechten Seite muß heißen $\exp \left(-E_{0} / k T\right)$.

S. 291, Gl. (38) muß heißen

$$
\begin{aligned}
& \Phi_{\text {schwach bewegl. }} \\
& \quad=(z-2) n^{2}(z-1)^{2}\left(\frac{n}{x}-1\right)[\exp (-\varepsilon / k T)-1] .
\end{aligned}
$$

S. 291, Gl. (42): Das letzte Glied der rechten Seite muß heißen

$$
\frac{p \Delta V_{2}}{\delta T}
$$

s. 292, Tab. 3, Sp. 3: In der Überschrift muß es heißen $10^{3}$.

s. 295, Abb. 2: In der Unterschrift muß es heißen

$$
\Delta H_{2}>0 \text {. }
$$

